\title{
Efficacy of Treatments for Depression in Children and Adolescents
}

\author{
Victoria A.J. Carr \\ Monash University, Australia \\ Candice P. Boyd \\ University of Ballarat, Australia
}

\begin{abstract}
Depression is a highly complex mental disorder that for many years was believed to be absent in children and adolescents. It is now accepted that depression not only exists in this age group, but also is a major mental health problem (Weller \& Weller, 2000a). Research suggests that the prevalence of depression in Australian children and adolescents is around 14\% (Boyd, Kostanski, Gullone, Ollendick, \& Shek, 2000). As depression appears to be highly prevalent, it is essential that efficacious treatments are identified, and that effective treatment strategies are established that best alleviate depressive symptoms in children and adolescents. In light of this need, this article details the criteria used to identify depression in children and adolescents, and examines the available evidence for the use of pharmacological and psychotherapeutic approaches in the treatment of depression in children and adolescents.
\end{abstract}

\section{The Symptoms of Depression}

Empirical research into the nature of depression has enabled the Diagnostic and Statistical Manual of Mental Disorders (DSM-IV-TR; American Psychiatric Association [APA], 2000) to detail the diagnostic criteria for children and adolescents. Clinicians often remember the main criteria by using the acronym DUMPS. This acronym covers childhood and adolescent manifestations of depression and includes most of the important DSM-IV-TR criteria (Carlson, 2000). Figure 1 details the components of the acronym DUMPS.

As indicated by Figure 1, the acronym DUMPS represents five of the criteria used to diagnose depressed children and adolescents. These criteria direct the clinician to examine the duration of the symptoms, the academic performance of the child, any physical ailments, and thoughts of pessimism and morbidity before making a diagnosis of depression (Carlson, 2000).
The manifestation of depression in children and adolescents can vary greatly with the developmental stage of the individual, with some symptoms of depression appearing to be more prominent at certain ages. For example, physical complaints, such as headache and stomach ache can arise in depressed individuals of any age; however, these symptoms have been shown to be evident in $100 \%$ of depressed preschool children (Carlson, 2000). In addition, recognising depression can be further complicated by the high rates of comorbidity (APA, 2000; Carlson, 2000; Weller \& Weller, 2000b). For example, depression in children and adolescents often co-occurs with anxiety disorders and disruptive behavioural disorders, such as attention deficit hyperactivity disorder and conduct disorder (APA, 2000). Given the complex nature of depression, and the variability in its expression, a variety of treatments have been developed whose efficacy continues to be examined. 


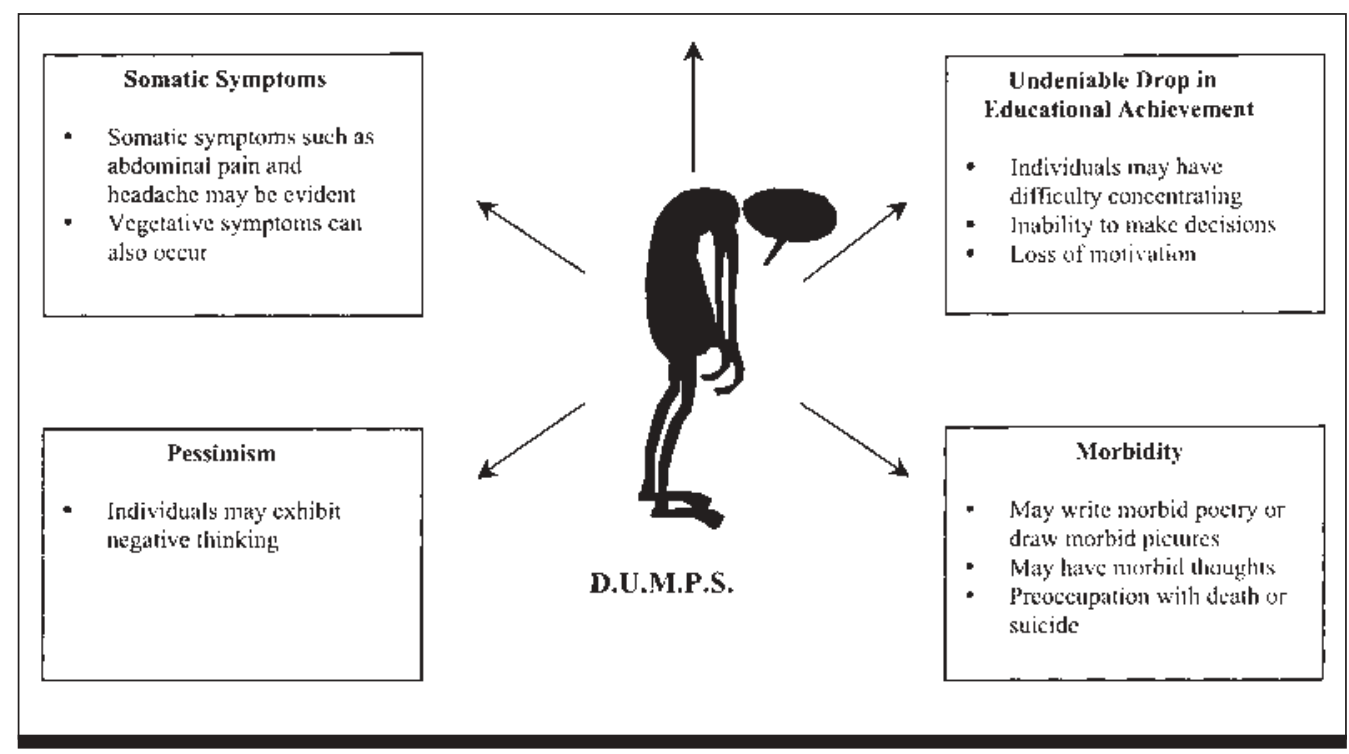

FIGURE 1

Manifestations of depression in children and adolescents.

\section{Treatments for Depression}

Treatment for depression falls broadly into two categories - pharmacological and psychotherapeutic. Of the two, pharmacotherapy is perhaps the most widely used treatment for depression in children and adolescents (Weller \& Weller, 2000b). The two main types of antidepressants used are the tricyclics (TCAs) and selective serotonin reuptake inhibitors (SSRIs), which act on the level of neurotransmitters operating within the brain. Research into the efficacy of TCAs has yielded largely negative results (Carlson, 2000; Weller \& Weller, 2000a). Michael and Crowley (2002) conducted a metaanalysis of 14 studies exploring the efficacy of TCAs as treatments for depression in children and adolescents (between 6 and 19 years of age). All studies were controlled clinical trials with active medication and placebo conditions. Results revealed that TCAs did not lead to a substantial reduction in depressive symptoms. In addition, it was found that individuals exhibited high responses to the placebo, which suggested that benefits were derived from expectations of the treatment, as opposed to the active medication. Similar research such as that by Hazell, O'Connell, Heathcote, Robertson, and Henry (1995) also suggested that TCAs are not substantially superior to placebos when treating depression in children and adolescents.

In contrast, in terms of the efficacy of SSRIs in treating depression in children and adolescents, research has yielded more positive results. Emslie and Mayers (1999) conducted one of the first studies into the efficacy of SSRIs and used a randomised study with 96 individuals between 7 and 17 years of age. Individuals either received a SSRI or placebo. After 5 weeks of treatment, the response to the SSRI was $56 \%$ compared with $33 \%$ for the placebo. In addition, no difference in the response rate between males and females or between prepubescent children and adolescents was found. Similarly, in a study of 45 participants with severe depression, most experienced complete remission by the end of the treatment period (mean $=8.4$ months; Rey-Sanchez \& Gutierrez-Casares, 1997). Overall, these studies suggest that SSRIs may be more effective than TCAs in treating depression in children and adolescents. Studies exploring SSRIs have found also that they have fewer side-effects than 
TCAs. While there appears to be conflicting evidence regarding the efficacy of pharmacological treatments of depression in children and adolescents, psychological approaches appear to possess more concrete and coherent evidence pertaining to their efficacy.

Within the broad category of psychotherapy, psychologists tend to rely on cognitive behavioural therapy (CBT) and interpersonal psychotherapy (IPT) when treating depression. These two therapies are widely used in treating depression in adults, and numerous studies have been conducted into the efficacy of these two therapies in treating depression in adult populations (Hollon, 1998). However, there appears to be a striking paucity of research into the efficacy of these therapies in treating child and adolescent depression (Carlson, 2000; Clarke, Rohde, Lewinsohn, Hops, \& Seeley, 1999; Mellin \& Beamish, 2002; Weisz, Thurber, Sweeney, Proffitt, \& LeGagnoux, 1997; Weller $\&$ Weller, 2000a). This lack of research is particularly alarming as it has been suggested that psychologists tend to adapt approaches for treating depressed adults and apply these treatments to depressed children and adolescents (Carlson, 2000; Weller \& Weller, 2000a).

The most common form of psychotherapy used to treat depression in children and adolescents is CBT (Curry, 2001). CBT is a structured form of psychotherapy directed at identifying and correcting erroneous beliefs and systematic distortions in an individual's thinking, in order to reduce distress and enhance the ability to cope (Blackburn, 1995; Hollon, 1998). Therapists use behavioural techniques at the beginning of treatment to alleviate any problem behaviours, such as indecisiveness, and poor concentration, which may prevent an individuals' progress. Following the implementation of behavioural treatments, training in cognitive techniques is undertaken to teach individuals more effective methods for dealing with the difficulties that contribute to their depression (Blackburn, 1995; Hollon, 1998).

Various studies have explored the efficacy of CBT with depressed children and adolescents (Weisz et al., 1997). Research on school-aged children comparing CBT with waitlist control groups revealed that individuals receiving CBT exhibited a significant reduction in depressive symptoms when compared to waitlist control groups (Clarke, et al., 1999; Weisz et al., 1997). In addition, studies have indicated that treatment gains resulting from CBT have been maintained at 5-, 10-, and 36-week follow-up periods (Butler, Miezitis, Friedman, \& Cole, 1980; Reynolds \& Coates, 1986; Weisz, et al., 1997). Similarly, Clark et al. (1999) explored the efficacy of CBT with 123 depressed adolescents aged between 14 and 18 years. Participants were randomly assigned to either CBT sessions with parent involvement, CBT without parent involvement, or a waitlist control group. Results indicated that both CBT groups yielded greater recovery $(66.7 \%)$ than the waitlist control group $(41.8 \%)$. When a follow-up was conducted 2 years later, both CBT groups did not differ in terms of the low recurrence of depression and the rates of recurrence were significantly lower than the waitlist control group.

Researchers have also compared the efficacy of CBT with other forms of psychotherapy such as problem solving therapy, supportive group therapy, systemic behavioural family therapy, and nondirective supportive therapy (Brent et al., 1997; Lerner \& Clum, 1990). For example, research by Brent et al. (1997) examined the efficacy of a number of psychotherapies with 107 depressed adolescents. Results indicated that $60 \%$ of individuals receiving CBT were in remission following the end of the treatment period. In contrast, only $38 \%$ of individuals receiving systemic behavioural family therapy, and 39\% receiving non-directive supportive therapy achieved remission. In addition, it was revealed that adolescents receiving CBT showed a more rapid reduction in depressive symptoms than individuals receiving the other types of psychotherapy. Overall, the findings from these studies suggest solid evidence that CBT is a successful treatment for depression in children and adolescents.

Despite the apparent efficacy of CBT as an effective treatment for depression, there are a number of limitations associated with these studies. Many studies have utilised small sample sizes, which may affect the significance of findings and may also impair the generalisability of findings to the wider population 
(Brent et al., 1997). Additionally, participants have been generally recruited through schools, rather than clinical settings, suggesting that findings may not be generalised to more severely affected clinical populations (Butler et al., 1980). Furthermore, many studies used a range of cognitive and behavioural interventions. Although these interventions have been based on cognitive-mediation models of depression, their emphasis varied, and little attention has been directed toward evaluating the specific CBT variables postulated to mediate improvement (Brent et al., 1997). Therefore, it is imperative that future research identifies the cognitive, interpersonal, and behavioural variables associated with the clinical changes reported. This would enable the identification of the particular aspects of CBT that cause changes in the levels of depression and it would assist in the refinement of CBT interventions. Furthermore, it is essential that more research into the efficacy of CBT generally as a method for treating depression in children and adolescents is undertaken as there is a paucity of research. Such research into the efficacy of CBT, which also accounts for the above limitations, would ensure that more accurate and generalisable findings are obtained.

The second treatment commonly used to treat depression in children and adolescents is interpersonal psychotherapy (IPT). IPT was originally developed for adult populations; however, it has been modified for use with children and adolescents (Moreau, Mufson, Weissman, \& Kleman, 1991). Interpersonal psychotherapy for adolescents (IPT-A) is a brief time-limited treatment which aims to reduce the symptoms related to depression by identifying the interpersonal problem areas connected with the beginning of the depression (Blanco, Lipsitz, \& Caligo, 2001; Moreau et al., 1991). No assumptions about the underlying origins of depression are hypothesised, rather the onset of depression is linked to one of four interpersonal problem areas (Mufson, Weissman, Moreau, \& Garfinkel, 1999; Mufson \& Fairbanks, 1996). These problem areas are interpersonal deficits, role transitions, interpersonal role disputes, and grief (Mufson et al., 1999). It is argued that determining the interpersonal problems that contribute to depression and working to alleviate those problems is sufficient in treating depression, regardless of other biological or personality factors (Blanco, 2001; Mellin \& Beamish, 2002; Moreau et al., 1991).

A limited number of efficacy studies have been conducted into the use of IPT-A as a treatment for depression in children and adolescents (Mufson \& Fairbanks, 1996; Mufson, Moreau, \& Weissman, 1994; Santor \& Kusumakar, 2001). For example, Mufson et al. (1994) conducted an open clinical trial with 14 depressed adolescents to test the efficacy of IPT-A. Adolescents attended 12 IPT-A therapy sessions conducted by a child psychologist. Results indicated a substantial reduction in depressive symptomatology and an improvement in general functioning. In addition, it was found that at the conclusion of the study, all participants had improved. In a follow-up study, Mufson and Fairbanks (1996) found that the treatment effects from the initial IPT-A therapy study were maintained over a 1-year period.

In a larger study Mufson et al. (1999) assessed the efficacy of IPT-A with 48 depressed adolescents. Results suggested that IPT-A was more effective in diminishing depressive symptomatology and increasing social functioning than clinical monitoring. Both clinician and self-reports suggested that adolescents receiving IPT-A reported a significantly greater decrease in depressive symptomatology and greater improvement in social functioning, such as relations with friends and dating. The drop out rate, however, in the clinical monitoring group was $54 \%$ as compared to $12 \%$ in the IPT-A group. The high attrition rate of the clinical monitoring group suggests that IPT-A may be more effective and appropriate in treating adolescent depression than clinical monitoring.

In addition, Rossello and Bernal (1999) investigated the effectiveness of IPT-A when compared with CBT and a waitlist control group. The study consisted of 71 Puerto Rican adolescents who met the diagnostic criteria for major depressive disorder and/or dysthymia. Both the IPT-A and CBT participant groups received 12 therapy sessions over 12 weeks. Results suggested that both IPT-A and CBT therapy sessions reduced depressive symptoms 
and increased self-esteem when compared with the waitlist control group. While neither IPT-A nor CBT were shown to be superior to each other, both were more effective in reducing depression when compared to the waitlist control group. This suggests that both IPT-A and CBT are effective treatments for depression in adolescence. Interestingly, it was also found that participants who received IPT-A had improved self-concept and social adaptation when compared with the waitlist control group. No differences were obtained for the CBT group and the waitlist control group for self-concept and social adaptation. These findings suggest that IPT impacts levels of functioning of a more interpersonal nature. This could be due to the theoretical basis of IPT, which purports to operate on interpersonal processes when reducing depression. However, they could also be due to IPT being a more suitable therapy to use with Puerto Rican communities, as their culture tends to place great importance on the notion of family and personal contact with others.

While evaluation studies of the efficacy of IPT-A have been promising, there are some methodological limitations, particularly sampling issues, that need to be considered. The majority of studies into the efficacy of IPT-A have utilised small sample sizes, which consisted of mostly female adolescents. Additionally, the majority of participants in these studies were from racial minority groups in the United States (Mufson \& Fairbanks, 1996; Mufson et al., 1994). Given the demographic makeup of participants, it is unclear whether the treatment effects are generalisable to other populations such as Caucasian male adolescents. In addition, some studies included participants that had comorbid diagnoses; therefore, complicating the ability to determine whether treatment effects are specific to the depression or another diagnosis. Other limitations include having no control group, using untrained individuals to administer IPT, and only conducting the therapy with one therapist (making it impossible to separate effects that are the result of the personal characteristics of the therapist rather than the therapeutic approach itself). These research design problems make treatment effects unclear, as it is difficult to determine whether treatment effects are due to the actual IPT-A treatment or other factors that were inherent in the research design (Mufson \& Fairbanks, 1996; Mufson et al., 1994; Mufson et al., 1999; Santor \& Kusumakar, 2001). In light of these limitations, the results of the studies into the efficacy of IPT-A as a treatment for child and adolescent depression must be viewed with caution.

Depression can be a serious and debilitating illness for adolescents and children. While the predominant treatment for depression continues be pharmacotherapy, a number of psychological treatments have been found to be effective. There is a lack of randomised controlled trials on the efficacy of CBT and IPT as treatments for child and adolescent depression. However, of the studies that do exist, it is apparent that both appear to be effective in alleviating depressive symptoms in children and adolescents. There are limitations associated with studies on both types of therapy that must be addressed in future research. Nevertheless, what is clear is that psychological approaches to the treatment of depression in children and adolescence suggest a viable and effective alternative or adjunct to the biological approach to treatment.

\section{References}

American Psychiatric Association. (2000). Diagnostic and statistical manual of mental disorders (4th ed., text rev.). Washington, DC: Author.

Blanco, C., Lipsitz, J., \& Caligor, E. (2001). Treatment of depression with a 12-week program of interpersonal psychotherapy. The American Journal of Psychiatry, 158, 371-375.

Blackburn, I.M. (1995). Cognitive therapy for depression and anxiety A practitioners guide. Oxford: Blackwell Science.

Boyd, C.P., Kostanski, M., Gullone, E., Ollendick, T.H., \& Shek, D.T. (2000). Prevalence of anxiety and depression in Australian adolescents: Comparisons with worldwide data. Journal of Genetic Psychology, 161, 479-492.

Brent, D.A., Holder, D.J., Birmaher, B., Baugher, M., Roth, C., et al (1997). A clinical psychotherapy trial for adolescent depression comparing cognitive, family, and supportive therapy. Archives of General Psychiatry, 54, 877-885.

Butler, L., Miezitis, S., Friedman, R., \& Cole, K.I. (1980). The effect of two school based intervention programs on depressive symptoms in pre-adolescents. Journal of American Educational Research, 17, 111-119. 
Carlson, G.A. (2000). The challenge of diagnosing depression in childhood and adolescence. Journal of Affective Disorders, 61, S3-S8.

Clarke, G., Rohde, P., Lewinsohn, P.M., Hops, H., \& Seeley, J.R. (1999). Cognitive-behavioural treatment of adolescent depression: Efficacy of acute group treatment and booster sessions. Journal of the American Academy of Child and Adolescent Psychiatry, 38, 272-279.

Curry, J.F. (2001). Specific psychotherapies for childhood and adolescent depression. Biological Psychiatry, 49, 1091-1100.

Emslie, G.J., \& Mayers, T.L. (1999). Depression in children and adolescents: A guide to diagnosis and treatment. CNS Drugs, 11, 181-189.

Hazell, P., O'Connell, D., Heathcote, D., Robertson, J., \& Henry, D. (1995). Efficacy of tricyclic drugs in treating child and adolescent depression: A metaanalysis. Bristish Medical Journal, 310, 897-901.

Hollon, S.D. (1998). What is cognitive-behavioural therapy and does it work? Current Opinion in Biology, 8, 289-292.

Lerner, M.S., \& Clum, G.A. (1990). Treatment of suicidal ideation. Behaviour Therapy, 21, 403-411.

Mellin, E.A., \& Beamish, P.M. (2002). Interpersonal theory and adolescents with depression: Clinical update. Journal of Mental Health Counseling, 24, $110-125$

Michael, K., \& Crowley, S.L. (2002). How effective are treatments for child and adolescent depression? A meta-analytic review. Clinical Psychology Review, $22,247-269$.

Moreau, D., Mufson, L., Weissman, M., \& Kleman, G.L. (1991). Interpersonal psychotherapy for adolescent depression: Description of modification and preliminary application. Journal of the American Academy of Child and Adolescent Psychiatry, 30, 642-651.

Mufson, L., \& Fairbanks, J. (1996). Interpersonal psychotherapy for depressed adolescents: A one-year naturalistic follow-up study. Journal of the American
Academy of Child and Adolescent Psychiatry, 35 , 1145-1155.

Mufson, L., Moreau, D., \& Weissman, M.M. (1994). Modification of interpersonal psychotherapy with depressed adolescents: IPT-A phase I and II studies. Journal of the American Academy of Child and Adolescent Psychiatry, 33, 695-705.

Mufson, L., Weissman, M.M., Moreau, D., \& Garfinkel, R. (1999). Efficacy of interpersonal psychotherapy for depressed adolescents. Archives of General Psychiatry, 56, 573-579

Reynolds, W.M., \& Coates, K.I. (1986). A comparison of cognitive-behavioural therapy and relaxation training for the treatment of depression in adolescence. Journal of Consulting and Clinical Psychology, 67, 734-745.

Rey-Sanchez, F., \& Gutierrez-Casares, J.R. (1997). Paroxetine in children with major depressive disorder: An open trial. Journal of the American Academy of Child and Adolescent Psychiatry, 36, 1443-1447.

Rossello, J., \& Bernal, G. (1999). The efficacy of cognitive-behavioural and interpersonal treatments for depression in Puerto Rican adolescents. Journal of Consulting and Clinical Psychology, 67, 734-745.

Santor, D.A., \& Kusumakar, V. (2001). Open trial of interpersonal therapy in adolescents with moderate to severe major depression: Effectiveness of novice IPT therapists. Journal of the American Academy of Child and Adolescent Psychiatry, 40, 236-240.

Weisz, J.R., Thurber, C.A., Sweeney, L., Proffitt, V.D., \& LeGagnoux, G.L. (1997). Brief treatment of mild to moderate depression using primary and secondary control enhancement training. Journal of Consulting and Clinical Psychology, 65, 703-707.

Weller, E.B., \& Weller, R.A. (2000a). Depression in adolescents: Growing pains or true morbidity. Journal of Affective Disorders, 61, S9-S13.

Weller, E.B., \& Weller, R.A. (2000b). Treatment options in the management of adolescent depression. Journal of Affective Disorders, 61, 23-28. 